\title{
$\square$ : Palinodie urbaine
}

\section{Henri-Pierre Jeudy}

Filósofo, professor e pesquisador do CNRS (Paris).

Autor das seguintes obras traduzidas no Brasil: Ardis da comunicação (Imago); O espelho das cidades

(Casa da Palavra); O corpo como objeto de arte

(Estação Liberdade); Ironia da comunicação (Sulina)

e Memória do social (Forense).

\section{Maria Claudia Galera}

Doutora em Literatura Comparada (USP) e pesquisadora (convidada) do CNRS (Paris). 

Sa petite maison est située au cœur de Sao Paulo. Elle est posée là, au milieu des buildings, comme une demeure incongrue. Par sa taille lilliputienne, elle ressemble à la maquette des habitations du siècle passé. Survivante des ruines que produisent les chantiers urbains, elle donne cette impression d'avoir été oubliée. Personne n'est venu la chercher pour l'emmener dans la lointaine périphérie où sa place serait plus convenue. C'est une petite femme au corps inachevé qui l'habite. La nuit, depuis le carreau brisé de sa fenêtre, elle observe la ville. Elle se poste derrière sa visière comme un guetteur cherchant entre les ombres nocturnes des immeubles les signes d'une vie aérienne. Nue, elle fait le pied de grue. Elle tremble en regardant au loin ce qui lui a toujours échappé. Elle suit la trajectoire d'un avion qui arrive des terres occidentales pour atterrir dans sa ville tropicale. Elle entend la musique monotone de ses réacteurs. Les cris de quelques noctambules retentissent. Et le silence fait retour. Elle ferme les yeux, elle voit les rues vides, les croisements perpendiculaires, les places, les rangées d'arbres, les hommes ou les femmes sans abris couchés sur les trottoirs, les rares véhicules qui passent à vive allure. Comme dans un rêve, elle est au cœur de la scène et reste au dehors. Elle se met à courir en ligne droite, sans jamais se soucier de bifurquer d'un côté ou de l'autre parce que les angles droits lui rappellent avec violence qu'il lui faudrait choisir une direction. Quand elle arrête un moment sa course pour reprendre son souffle, elle passe ses mains sur la peau humide de son ventre. Sans bouger, elle attend que la ville entre en elle. Elle lève les bras, elle tend les jambes, elle appuie tout son corps contre un mur, elle devient le mur.

Et au-delà du mur, elle aperçoit l'autre ville, la ville rêvée. Maintenant, elle est à Paris. Lentement, elle s'éveille, elle demeure un instant ébahie, les yeux grands ouverts, elle se met à marcher sur les boulevards, se laisse conduire par les rues mythiques, retrouve les endroits légendaires, captée par les images dont elle s'est toujours servi pour effacer la ville réelle, celle qui chaque jour s'impose à son regard. Elle se cent elle-même si petite en face de ses songes 
qui deviennent si réels. Elle marche sans but, il lui faut se perdre pour refaire la connaissance de ce territoire déjà connu d'après les cartes postales en blanc et noir, les plans vieillis et jaunes et surtout les anciennes chansons qui lui ont permis d'imaginer toutes les guinguettes que jamais le temps n'a fait disparaître. Elle voit le Paris du XIXème siècle, la foule qui déambule, les femmes aux vieux chapeaux avec leurs coiffures d'autrefois, les hommes en frac avec leurs cannes aux pommeaux en corne, avec leurs moustaches recourbées. A Sao Paulo, les miroirs des buildings ne réfléchissent jamais les visages des gens qui passent. Il y a une guerre invisible qui fait tomber le passé sans ériger le présent, il y a ce délire du renouvellement qui fait qu'on ne sait jamais ce qui est en train de se faire, de se refaire ou de se défaire.

Chaque nuit, elle traverse l'Atlantique pour rejoindre Paris. Elle quitte la ville chantier, là où les ruines se confondent avec les dernières constructions. A bout de souffle, elle s'arrête de temps à autre dans les bistrots, elle goûte du vin rouge comme si elle buvait un breuvage divin, un nectar qui pourrait la rendre immortelle, - il semble l'avoir déjà fait pour tous ces gens anachroniques qui si baladent dans les rues -. Elle boit la ville pétillante avec son regard. Point de convergence des fleuves et des routes, ville qui coule au bord de la Seine, rive gauche, rive droite, tous les chemins de son imagination la ramènent à Paris. Le rouge et le noir. Elle pleure pour les incendies, pour la peste et le choléra, elle voit les dames somnambules sur les balcons, elle observe un jeune homme aux cheveux verts qui allume une cigarette en face du Panthéon. Les temps s'accumulent, ils ne s'annulent pas. Des couches et des couches de pierres taillées. Elle marche dans une rue dont elle ne connaît pas le nom, elle tombe sur les traces du plus vaste monument de l'Occident médiéval, sur une église gothique, sur un palais colossal. Elle change de trottoir, les bruits de la ville font des échos dans sa peau, elle a des frissons partout. Les faubourgs contre l'érection de l'enceinte, les barricades contre les rois, La Bastille contre Versailles, les feux de la Commune, les têtes tranchées qui sont encore à terre, les bruits d'antan qui 
se mélangent aux voix d'aujourd'hui, du jamais, du rien, elle entend les musiques et les cris, elle absorbe tout ce qu'elle voit pour revenir le matin à Sao Paulo, dans sa ville sans mémoires vives.

La ville transpire, la ville est capricieuse, elle ne se donne pas si facilement, surtout la nuit lorsqu'elle devient reine des simulacres. Les signes qu'elle délivre au grand jour s'évanouissent. Ne reste que l'ombre des formes immobiles de l'enchevêtrement tentaculaire des buildings. Il y a bien la lune, en croissant moqueur, qui éclaire les hauteurs invisibles. Voilée par le mouvement des nuages, elle semble danser sa danse macabre. La ville fait naître l'autre ville au loin, la ville rêvée, la ville à laquelle son corps inachevé a donné la souveraineté d'un mythe. Immatérielle, la ville demeure présente dans les images des citadins qui la transforme et l'embellisse grâce à des souvenirs qui ne leur appartiennent pas, des souvenirs débridés qui se glissent au fil du temps dans une construction d'un récit sans fin, toujours en train de tracer les signes de son origine. Subreptices, ces retours de mémoire surgissent au détour d'une promenade ou d'un parcours mille fois recommencé, bousculant en douceur l'ordre répétitif des habitudes. Et rien, pas même les nouveaux chantiers, ne vient s'opposer à leur étrange actualité. Ils n'ont plus besoin, ces retours de mémoire, de se mesurer à une quelconque vérité, ils s'engendrent en toute liberté, forgeant la trame confuse des temps anciens. Toute ville demeure pourtant souriante aux autres villes du monde. Toute ville fait sombrer dans l'oubli ses fébrilités diurnes pour imposer au regard insomniaque le souvenir d'un autre temps. Mémoires vacillantes.

Retour sur le chaos urbain qui devient étrangement une figure d'ordre. Les corps du dimanche dans les avenues momentanément sans véhicules. Bicyclettes, patins à roulettes, skate-board... Footing sur les chaussées abandonnées à la courbure des voûtes plantaires. Les affiches à voir, les affiches vues et revues, de l'embouteillage à la promenade pédestre. Les gros seins et les grosses fesses, modèles anatomiques imposés comme 
le destin obligé au devenir de la femme fatale. Confieznous votre argent, nous ne le vous rendrons pas après l'avoir fait fructifier. C'est le nouveau jeu du capitalisme avec les vieux. Du suspense! Après ses hallucinations parisiennes de la nuit, sa ville ne lui semble plus pareille. Elle regarde les rues qu'elle connaît depuis toujours, les trottoirs abîmés, les fils électriques qui tranchent le ciel, elle ne peut pas marcher comme avant, les trajets plusieurs fois répétés se sont effacés de son corps, ses gestes accomplis sans réflexion, ses automatismes ne se fondent plus dans l'allure pressée des Paulistes. Malgré la musique éternellement dissonante, les klaxons nerveux, les voitures rendues impatientes par la lenteur des feux rouges, le bruit des moteurs, les cris étouffés par la densité béton, elle entend le silence glacial de l'architecte dans la raideur des façades. Le cauchemar urbain se révèle à la lumière du rêve d'une ville toujours réinventée.

Assise sur un strapontin dans le métro, comme elle pourrait l'être au cinéma quand il n'y a plus de place, elle ferme les yeux, et la ville au-dessus abandonne son opacité pour prendre une forme de plus en plus diaphane. La ville transparente, pressentie depuis la pénombre du dessous, la ville éblouie par la lumière du soleil. Recroquevillée sur son strapontin, elle imagine une ville toute en verre qui lui renverrait en miroir des myriades d'images de son corps afin de le faire disparaître, happé par la luminescence. Le paradis futuriste des architectes frappés par l'hystérie du verre, construisant des villes sans regard, des villes qui chassent le regard quand tout est tellement donné à voir que la vision elle-même n'a plus lieu d'être. Elle ouvre alors les yeux, observe les gens qui montent ou qui descendent, se laisse captée par la promiscuité des corps pour retrouver l'impression de la densité. La panoplie des attitudes, le mouvement suspendu dans l'attente de la prochaine station, les gestes esquivés, la main sur les tubes, dans les anneaux, le dos appuyé contre les portes, les visages souriants, crispés, soucieux, les paroles inaudibles, les paroles murmurées dans l'oreille, et parfois une adresse folle aux gens, cette adresse de 
quelqu'un qui, à n'importe quelle heure de la journée, prend à témoin le genre humain.

Quand elle regarde, depuis la fenêtre de sa maison, les hélicoptères qui se posent sur la plate-forme, en haut d'un building, elle pense à tous ces gens riches qui, passant de leurs villas protégées comme des châteaux du Moyen Age occidental aux lieux des affaires économiques, ignorent désormais la ville, devenue, pour eux, le territoire d'un désastre permanent. Ils quittent leur condominium pour se faire de l'argent sur le dos d'une plèbe miséreuse qu'ils ne veulent plus apercevoir. Ils survolent le paysage insalubre de la ville tentaculaire qui, en dessous d'eux, poursuit son aventure catastrophique. Ils n'osent pas avouer qu'ils envisagent parfois de voir liquider les favelas au napalm. Tous ces foyers de guérilla urbaine qui menacent la tranquillité de ceux qui font fructifier leur fortune. La sécurité urbaine a un prix, celui de l'élimination des chancres qui font de la ville une poubelle humaine explosive. Voilà ce à quoi ils songent, chaque jour, ces gens-là, en volant audessus de ce qui, pour eux, n'est déjà plus une ville.

Lorsqu'elle remonte vers sa petite maison, par une ruelle pavée, elle a toujours cette impression d'entrer dans un lieu historique. Il y a d'autres maisons du même genre que la sienne, des arbres fleuris, une place minuscule sur la gauche, et surtout de l'herbe pousse entre les pavés comme si la nature tropicale restait prête à reconquérir son territoire. Ces habitations ont été construites dans les années 50 , elles ne sont pas très anciennes, elles étaient là avant les buildings qui maintenant les entourent. Elles représentent pourtant un fragment d'histoire d'une ville qui recommence indéfiniment son histoire. Sao Paulo chasse son propre passé. Toutes les villes, même les plus récentes, gardent pourtant un passé. Mais Sao Paulo semble s'acharner à rejeter ses mémoires citadines. Les Paulistes aiment à dire que, du jour au lendemain, ils peuvent ne plus reconnaître le lieu où ils habitent, comme si, dans la nuit, les démons de la métamorphose urbaine avaient encore frappé. Elle, au moment où elle approche de sa maison, elle s'autorise à croire qu'elle vit dans les derniers 
restes d'un décor patrimonial bien que ce lieu exigu de la ville n'est qu'un passé très récent. Un passé qui s’impose comme une entrevue atemporelle puisqu'il y a trop peu de temps pour qu'il puisse paraître historique. De l'histoire récente, mais de l'histoire tout de même. Les noms de rue. Jupiter, Urano, Esmeralda, Alabastro... En rejoignant son quartier, elle découvre son lieu de naissance, elle repère des places, des statues dans la rue qui, bien souvent, lui étaient invisibles, elle se laisse aller sans but dans $\mathrm{Acli}$ mação. Elle se rappelle être venue quelques fois dans ce parc. Souvenir joyeux de jardins symétriques, de compositions aux ambitions esthétiques inimaginables. Elle marche au bord du lac, elle s'assied, et retrouve la réplique du parc d'Acclimatation parisien. À São Paulo, il y a plusieurs endroits comme celui-là, on reproduit des lieux parisiens, le Trianon, la Place de la Concorde, les Champs Elysées. Puis, au fil du temps, tout se transforme, le centre devient périphérie, la ville se développe comme un cancer, la ville se déchire et s'engendre, les noms des lieux sont dévorés, les formes et les fonctions sont transfigurées. Dans ce parc, il y avait des animaux. Selon la rumeur qui circule encore, les fonds destinés à la manutention du parc ont été détournés, les résidants du voisinage se sont occupés des animaux. Un beau jaguar a fini ses jours dans le jardin d'une grande maison qui a résisté à la fureur de l'industrie du bâtiment. Une vieille dame qui n'était pas soumise à la quête d'une jeunesse perpétuelle, a gardé ses beaux cheveux blancs, son chignon magnifique, elle roulait dans les rues du quartier dans une voiture des années cinquante. C'est elle qui a pris soin du jaguar jusqu’à sa mort.

La ville garde sa part d'invisibilité. L'exhibition publique de ses myriades de signes n'anéantit jamais ses secrets. Ses lieux les plus fréquentés demeurent aussi des lieux dérobés au regard. Eternel territoire de la clandestinité, elle laisse à quiconque la possibilité de faire corps avec elle. De ses innombrables recoins, de ses places au grand jour, elle apaise les angoisses, chasse les amertumes du promeneur ivre qui, au petit matin, cherche 
en vain à retrouver son domicile. Elle présente aux amants en peine ses bancs et ses bords de trottoir, ses marches d'escalier, elle procure aux clochards la chaleur de ses bouches de métro. Jamais les récits qui multiplient ses images, ses clichés, n'épuiseront sa puissance à faire naître ce sens ultime d'être au monde quand s'effondre l'ordre usuel des représentations. Et celui qui, à l'aube encore, décidé de mettre un terme à sa vie, choisit avec pudeur l'espace public pour passer à l'acte, offre le sacrifice de son corps à la ville.

Il n'y a pas de ville qui se donne à sentir et à voir sans mémoire d'autres villes. Qu'elle subisse la turbulence de ses mutations rapides ou qu'elle se drape dans la magnificence de son passé, la ville construit son hors temps. Que persistent les figures de son anachronisme, que celles-ci surgissent au cœur de sa modernité, la ville crée ses propres effets de disruption et de circonvolution temporelles. Source de mémoires inachevées, la ville se dérobe autant à sa conservation patrimoniale qu'à sa rapide métamorphose. Les morts côtoient les vivants. Et si les morts revenaient, ils envahiraient les rues, ils provoqueraient un gigantesque embouteillage, ils tiendraient des propos incohérents. Quand ils sont là, derrière notre dos, ils n'arrêtent pas de rire, un rire comme un râle qui ne pue pas vraiment, on aurait imaginé une haleine plus fétide, le mort qui sent bon est fréquentable, il donne l'impression de se parfumer pour sortir de son trou, une courtoisie qui fait plaisir, le mort pourrait être hargneux, il est là, debout entre nous, il part en visite, il est heureux de nous voir, d'ailleurs il le dit en faisant " pfuit, pfuit, pfuit », juste trois fois, pour signaler qu'il ne prendra pas trop de place.

Dans la chambre de sa petite maison à Sao Paulo, elle vient de rêver qu'elle doit aller à la morgue de Paris reconnaître son corps. Elle ne sait pas si elle réussira à le faire, même s'il lui ressemble. Elle est très inquiète, elle n'a pas encore réalisé qu'elle était morte. Elle continue à vivre comme si rien ne s'était passé. Il faut qu'elle voit son corps mort. A la morgue, le préposé à la garde des 
macchabées ne trouve pas le tiroir dans lequel son corps devrait être, il tire les tiroirs l'un après l'autre, il soulève les draps pour voir les têtes. Elle n'est pas là. Dans un tiroir vide, le drap froissé indique bien qu'il devait y avoir un cadavre. Le préposé se souvient qu'il l'avait placé là au début de la matinée. Il dit : où a-t-il pu aller? Elle sourit, elle n'est jamais là où on l'attend. Avant que le jour se lève, elle reprend sa marche dans Paris. À cette heure-là, la ville dort encore. Capricieuse, elle cache ses véritables formes, celles qui lui donnent l'allure qu'elle a prise au fil des siècles, elle se refuse encore au jeu matinal de son dévoilement, elle s'amuse en imposant au regard l'instabilité vague de ses ombres ondoyantes. L'air frais de la première lueur de l'aube ne va pas tarder, sa promenade la conduit, cette fois-ci, vers un endroit précis, ses pas redécouvrent le chemin d'après les souvenirs que des cartes pour touristes lui ont laissés, ses yeux sont touchés par les rues et les façades comme les doigts de l'aveugle qui ne devine les formes que par ses caresses. Elle ne met pas très longtemps pour arriver du côté Est de Paris. La sueur perlant sur son visage, elle entend les palpitations de son propre bâtiment cardiaque. Le peu de gens dans la rue à cette heure-là, ce sont des hommes et des femmes habillés en noir. Vingtième arrondissement, Ménilmontant, elle entre au cimetière du Père-Lachaise. La ville cumule ses deuils, les vivants rendent hommage à leurs morts. Elle sent la peau froide de chaque marche lorsqu'elle monte l'escalier. Il fait encore plus frais. Les arbres séculaires, le marbre mortuaire et partout, cette puissance des histoires de vie des morts. Elle ralentit sa marche, elle se laisse retenir doucement par les épitaphes, les photographies des morts, les pots de fleurs par terre, la pierre, la pierre froide et les inscriptions lapidaires. Dans le silence sépulcral, elle capte la musique des prières du passé, les lamentations des anciens vivants pour leurs morts. Elle cherche certaines tombes, elle en découvre de nouvelles, le plan du cimetière n'est pas encore présent à son esprit comme celui de la ville des vivants. les mots de la langue pour les morts lui reviennent, les corbillards, 
les tombeaux, les cercueils... Les corps des fantômes qui peuplent ses jours depuis toujours se trouvent là, les uns à côté des autres en face de ses yeux. Pétrifiés, il se putréfient depuis longtemps. Et c'est dans cet état qu'ils se donnent à son regard, les légionnaires de l'absinthe, la mélancolie et la joie des maudits, le génie et le désespoir des poètes, les cris de la mort en vie et cette tombe de Miguel gel Asturias, écrivain guatemaltéque, artisan du métissage culturel, qui a lui-même choisi d'être inhumé au Père-Lachaise. Elle est venue déposer des fleurs sur les tombes de ses funestes amis, elle est venue leur offrir des bouquets de maracudja, fruits de la passion, fleurs du sommeil, ce cadeau léthargique pour partager avec eux sa torpeur exotique. Les lumières du jour pointent, elle doit partir, puisque de l'autre côté de l'océan, la nuit arrive, elle ne peut pas être en retard. Elle marche en suivant des voies étroites, l'herbe pousse davantage dans certains coins oubliés par les vivants, elle cherche le mur qu'elle doit traverser pour retrouver sa petite maison de l'autre côté de l'Atlantique. Avant de partir, les fusillades des derniers communards la retiennent quelques instants, il y a du sang qui coule par terre. Elle croise le mur des fusillés, de l'autre côté il y a aussi des coups de feu, du sang par terre, les corps des morts sont encore chauds. Au-delà du mur, une autre guerre est en cours.

De retour là-bas, elle songe à la ville des morts éternels, à la ville où les morts sont si présents que les vivants ne sont jamais en deuil. Même les inconnus y trouvent leur nom. Elle, elle construit sa propre histoire là-bas, à Paris pour la ramener ici, à Sao Paulo, comme cet ailleurs qui lui donne son inscription sur un territoire sans nom. Le perpétuel voyage dans l'épaisseur d'un hors temps qui fait de l'anonymat urbain la possibilité de toujours se nommer. 